Pacific Journal of Mathematics

THE CONGRUENCE EXTENSION PROPERTY FOR COMPACT 


\title{
THE CONGRUENCE EXTENSION PROPERTY FOR COMPACT TOPOLOGICAL LATTICES
}

\author{
Albert R. StralKa
}

\begin{abstract}
Let $L$ be a compact, distributive topological lattice of finite breadth and let $A$ be a closed sublattice of $L$. It is shown that every closed congruence on $A$ can be extended to a closed congruence on $L$. An example is provided to show that the requirement of finite breadth cannot be deleted.
\end{abstract}

The congruence extension property serves to characterize distributive lattices (cf. [4]). The definition of this property may be reformulated for topological lattices as follows: A topological lattice $L$ has the congruence extension property if given any closed sublattice $A$ of $L$ and any closed congruence $[\varphi]$ on $A$ there is a closed congrucence $[\Phi]$ on $L$ such that $[\Phi] \cap(A \times A)=[\varphi]$. When this situation prevails we say that $[\varphi]$ has been extended to a closed congruence $[\Phi]$ on $L$. In this paper we prove that compact, distributive lattices of finite breadth have the congruence extension property. This fact is established by first showing that the lattice of closed congruences for such lattices is distributive. We also prove that the compact topological lattice $X=X_{i=1}^{\infty}\{0,1\}$ with coordinatewise operations does not have the congruence extension property.

1. Preliminaries. A finite subset $B$ of a lattice is meet-irredundant if no proper subset of $B$ has the same meet as $B$. The breadth of a lattice $L, B r(L)$, is the supremum of the cardinalities of its meet-irredundant sets. A chain is a lattice whose breadth is one. An element $p$ of a lattice is prime if $x \wedge y \leqq p$ implies that $x \leqq p$ or $y \leqq p$. We shall use the notation that if $[\varphi]$ is a congruence then $\varphi$ is the canonical map associated with $[\varphi]$.

A topological lattice is a Hausdorff topological space with a pair of continuous maps $\wedge, \vee: L \times L \rightarrow L$ such that $(L, \wedge, \vee)$ is a lattice. A point $p$ of a subset $A$ of a topological lattice $L$ is a local maximum of $A$ if there is an open subset $U$ of $L$ such that ( $U \cap$ $A) \cap(p \vee L)=\{p\}$. By $A^{*}$ we shall mean the topological closure of $A$.

Suppose that $L$ is a compact topological lattice. $\mathscr{C}(L)$ is the lattice of closed congruences on $L$ (considered as subsets of $L \times L$ ) with operations $\wedge$ and $\vee$ defined by $[\varphi] \wedge[\theta]=[\varphi] \cap[\theta]$ and $[\varphi] \vee[\theta]$ is the smallest closed congruence on $L$ which contains both $[\varphi]$ and $[\theta]$. $\mathscr{L}(L)=\{[\varphi] \in \mathscr{C}(L) ; B r(\varphi(L))=1\}$. For $[\varphi] \in \mathscr{L}(L)$ we define $M(\varphi)=\left\{x \in L ; x=\vee \varphi^{-1}(\varphi(x))\right\}$ and $m(\varphi)=\left\{x \in L ; x=\wedge \varphi^{-1}(\varphi(x))\right\}$. 
Since $\varphi(L)$ is a chain both $M(\varphi)$ and $m(\varphi)$ are chains. Also since $\varphi(L)$ has the order topology when $M(\varphi)$ and $m(\varphi)$ are endowed with the order topology they are homeomorphic and isomorphic with $\varphi(L)$. Associated with $\varphi$ there are two natural (algebraic) homomorphisms $\varphi^{1}$ and $\varphi^{0}$ where $\varphi^{1}(x)=\varphi^{-1}(\varphi(x)) \cap M(\varphi)$ and $\varphi^{0}(x)=\varphi^{-1}(\varphi(x)) \cap m(\varphi)$.

We say that a collection $\mathscr{P}$ of disjoint closed intervals of a compact topological lattice $L$ is a partition of $L$ if (a) $\cup \mathscr{P}=L$ and (b) $P_{1}, P_{2} \in \mathscr{P}$ implies that $P_{1} \wedge P_{2} \subseteq P_{1}$ or $P_{1} \wedge P_{2} \subseteq P_{2}$. To every partition of $L$ there corresponds a member of $\mathscr{L}(L)$. For $[\varphi] \in \mathscr{L}(\mathrm{L})$ we shall let $\mathscr{P}_{[\varphi]}$ denote the partition of $L$ corresponding to [ $\left.\varphi\right]$. It is easily proved that if $[\varphi]$ and $[\theta]$ are in $\mathscr{L}(L)$ then $\mathscr{P}_{[\varphi] \vee[\theta]}=$ $\varphi^{-1}\left(Q^{\prime}\right)$ where $Q$ is the smallest partition on $\varphi(L)$ which contains $\varphi\left(\mathscr{P}_{[\theta]}\right)$.

Recall that a coordinate chain in a lattice $L$ is a chain $C$ such that (1) $C$ consists only of prime elements of $L$ and (2) $C$ is closed with respect to arbitrary meets [2]. Note that if $L$ is a compact topological lattice (2) is equivalent to $\left(2^{\prime}\right) C$ is closed with respect to decreasing nets.

Lemma 1.1. Let $L$ be a compact topological lattice and let $[\varphi] \in$ $\mathscr{L}(L)$. Then $M(\varphi)$ is a coordinate chain.

Proof. Let $a \in M(\varphi)$ and suppose that for some pair $x, y \in L, x \wedge$ $y \leqq a$. Since $\varphi(L)$ is a chain and $\varphi$ is a homomorphism

$\varphi(a) \geqq \varphi(x \wedge y)=\varphi(x) \wedge \varphi(y)=\min \{\varphi(x), \varphi(y)\}$.

Let $\varphi(x)=\min \{\varphi(x), \varphi(y)\}$. Because $a \in M(\varphi)$ we have $a \geqq x$. Thus $a$ is a prime element of $L$.

Let $A$ be a decreasing net of elements of $M(\varphi)$. Since $L$ is compact $A$ converges to some element $a_{0} \in L$. Suppose that for some pair $x, y \in L, a_{0} \geqq x \wedge y$. Then $a \geqq x \wedge y$ for each $a \in A$. Since $a$ is prime either $a \geqq x$ or $a \geqq y$. Thus we may assume that there is a cofinal subnet $A^{\prime}$ of $A$ such that for each $a \in A^{\prime}, a \geqq x$. Hence $a \wedge$ $x=x$ for all $a \in A^{\prime}$ and by the continuity of $\wedge, a_{0} \wedge x=x$. Therefore $a_{0}$ is a prime element of $L$. Since $A^{\prime}$ is a decreasing net and it converges to $a_{0}$ it follows that $a_{0} \in M(\varphi)$.

\section{The congruence extension property on compact lattices.}

LEMMA 2.1. Let $L$ be a compact topological lattice and let $[\alpha]$, $\left[\beta_{1}\right],\left[\beta_{2}\right], \cdots,\left[\beta_{n}\right] \in \mathscr{L}(L)$. Then $[\alpha] \vee\left(\bigwedge_{i=1}^{n}\left[\beta_{i}\right]\right)=\bigwedge_{i=1}^{n}\left([\alpha] \vee\left[\beta_{i}\right]\right)$.

Proof. To simplify notation we shall let $[\varphi]=[\alpha] \vee\left(\bigwedge_{i=1}^{n}\left[\beta_{i}\right]\right)$. Note that since $[\varphi] \geqq[\alpha]$ we must have $[\varphi] \in \mathscr{L}(L)$. It is always 
the case that $[\varphi] \leqq \Lambda_{i=1}^{n}\left([\alpha] \vee\left[\beta_{i}\right]\right)$ so we need only prove that $[\varphi] \geqq$ $\bigwedge_{i=1}^{n}\left([\alpha] \vee\left[\beta_{i}\right]\right)$. This will be done by showing that if $(x, y) \notin[\varphi]$ then there is $i \in\{1,2, \cdots, n\}$ such that $(x, y) \notin[\alpha] \vee\left[\beta_{i}\right]$ and consequently $(x, y) \notin \bigwedge_{i=1}^{n}\left([\alpha] \vee\left[\beta^{i}\right]\right) . \quad(x, y) \notin[\varphi]$ implies that $\varphi^{1}(x) \neq \varphi^{1}(y)$. We may assume that $\phi^{1}(x)<\phi^{1}(y)$.

(1) If $a, b \in M(\varphi)$ with $a<b$ then there is $i \in\{1,2, \cdots, n\}$ such that $\beta_{i}(a)<\beta_{i}\left(\varphi^{0}(b)\right)$.

If this were not the case then for each $i \in\{1,2, \cdots, n\}$ we would have $\beta_{i}^{1}\left(\varphi^{\circ}(b)\right) \leqq \beta_{i}^{1}(a) \leqq \beta_{i}^{\prime}(b)$. This implies that

$$
\varphi^{0}(b) \leqq \bigwedge_{i=1}^{n} \beta_{i}^{\prime}\left(\varphi^{0}(b)\right) \leqq \bigwedge_{i=1}^{n} \beta_{i}^{\prime}(a) \leqq \bigwedge_{i=1}^{n} \beta_{i}^{\prime}(b) .
$$

Then because $a \leqq \Lambda_{i=1}^{n} \beta_{i}^{\prime}(a) \leqq \beta_{i}^{l}(a)$ for $i=1,2, \cdots, n$ we have (a, $\left.\bigwedge_{i=1}^{n} \beta_{i}^{1}(a)\right) \in \bigwedge_{i=1}^{n}\left[\beta_{i}\right] . \quad$ For the same reason $\left(\varphi^{0}(b), \bigwedge_{i=1}^{n} \beta_{i}^{\prime}\left(\varphi^{0}(b)\right)\right)$, $(b$, $\left.\bigwedge_{i=1}^{n} \beta_{i}^{\prime}(b)\right) \in \bigwedge_{i=1}^{n}\left[\beta_{i}^{1}\right]$. Then since $\left(\varphi^{0}(b), b\right) \in[\varphi]$ and $\varphi^{0}(b) \leqq \bigwedge_{i=1}^{n} \beta_{i}^{l}(a) \leqq$ $\bigwedge_{i=1}^{n} \beta \bigwedge_{i}^{1}(b)$ we have $\left(b, \Lambda_{i=1}^{n} \beta_{i}^{l}(a)\right) \in[\varphi]$. Hence $(a, b) \in[\varphi]$. This is a contradiction so there must be an $i \in\{1,2, \cdots, n\}$ with the property that $\beta_{i}(a)<\beta_{i}\left(\varphi^{0}(b)\right)$.

Suppose that $\varphi\left(\left[\varphi^{1}(x), \varphi^{1}(y)\right]\right)$ is not connected then because $\varphi(L)$ is a chain there are $a, b \in M(\phi)$ such that $\varphi^{1}(x) \leqq a<b \leqq \phi^{1}(y), L=$ $(a \wedge L) \cup\left(\varphi^{\circ}(b) \vee L\right)$ and $\varphi(L)=\varphi(a \wedge L) \cup \varphi\left(\varphi^{\circ}(b) \vee L\right)$. From (1) above there is $i \in\{1,2, \cdots, n\}$ such that $\beta_{i}(a)<\beta_{i}\left(\varphi^{0}(b)\right)$. This implies that $\beta_{i}(L)=\beta_{i}(a \wedge L) \cup \beta_{i}\left(\varphi^{0}(v) \vee L\right)$ and $\beta_{i}(a \wedge L) \cap \beta_{i}\left(\varphi^{\circ}(b) \vee L\right)=$ $\varnothing$. It then follows that $(a, b) \notin[\varphi] \vee\left[\beta_{i}\right]$. Then because $[\alpha] \leqq[\varnothing]$, $x \leqq \varphi^{1}(x) \leqq \mathrm{a}$ and $\varphi^{0}(b) \leqq \varphi^{0}(y) \leqq y$, we must have $(x, y) \notin[\alpha] \vee\left[\beta_{i}\right]$.

Thus we have disposed of the case in which $\varphi\left(\left[\varphi^{1}(x), \varphi^{1}(y)\right]\right)$ is not connected so we may assume throughout the rest of the proof that $\varphi\left(\left[\varphi^{1}(x), \varphi^{1}(y)\right]\right)$ is connected.

(2) If $a \in M(\varphi) \cap\left[\varphi^{1}(x), \varphi^{1}(y)\right] \backslash\left\{\varphi^{1}(y)\right\}$ then there is $i \in\{1,2, \cdots, n\}$ such that if $c \in M(\varphi)$ and $a<c$ then $\beta_{i}(a)<\beta_{i}\left(\varphi^{0}(c)\right)$.

Since $\varphi\left(\left[\varphi^{1}(x), \varphi^{1}(y)\right]\right)$ is connected a cannot be a local maximum of $M(\varphi)$. Hence there is a net $B$ of elements of $M(\varphi)$ which converges to $a$ such that if $b \in B$ then $a<b \leqq \varphi^{1}(y)$. Using (1) above and the fact that $n$ is finite we may find an element $i \in\{1,2, \cdots, n\}$ and a cofinal subnet $B^{\prime}$ of $B$ such that if $b \in B^{\prime}$ then $\beta_{i}(a)<\beta_{i}\left(\varphi^{\circ}(b)\right)$. Then if $c \in M(\varphi)$ and $c>a$ there must be $b \in B^{\prime}$ with the property that $a<$ $b \leqq c$. Thus $\beta_{i}(a)<\beta_{i}\left(\varphi^{0}(b)\right) \leqq \beta_{i}\left(\varphi^{0}(c)\right)$.

(3) There is $i \in\{1,2, \cdots, n\}$ and $a, b \in M^{\prime}(\varphi) \cap\left[\varphi^{1}(x), \varphi^{1}(y)\right]$ with $a<b$ such that if $c, d \in M(\varphi)$ and $a \leqq c<d \leqq b$ then $\beta_{i}(c)<$ $\beta_{i}\left(\varphi^{0}(d)\right)$.

For $s \in M(\varphi) \cap\left[\varphi^{1}(x), \varphi^{1}(y)\right]\left\{\varphi^{1}(y)\right\}$ we let $\xi(s)$ be any element of $\{1$, $2, \cdots, n\}$ with the the property that if $t \in M(\varphi)$ and $s<t$ then $\beta_{\xi(s)}(s)<\beta_{\xi(s)}\left(\varphi^{0}(t)\right)$. From (2) above $\xi$ is a function defined for each element of $M(\varphi) \cap\left[\varphi^{1}(x), \varphi^{1}(y)\right] \backslash\left\{\varphi^{1}(y)\right\}$. Let $A_{i}$ be the closure of $\xi^{-1}$ (i) in $M(\varphi)$ when $M(\varphi)$ is endowed with the order topology. With this 
topology $M(\varphi) \cap\left[\varphi^{1}(x), \varphi^{1}(y)\right]$ becomes a compact connected chain. Hence some $A_{i}$ must contain a nondegenerate interval of $M(\varphi) \cap$ $\left[\varphi^{1}(x), \varphi^{1}(y)\right]$. Let that interval be $[e, f] \cap M(\varphi)$. Then, still using the order topology on $M(\varphi), \xi^{-1}(i)$ is dense in $[e, f]$. Hence there are $a, b \in \xi^{-1}(i)$ with $e \leqq a<b \leqq f$. $\quad \xi^{-1}(i)$ is dense in $[a, b] \cap M(\varphi)$ so by an argument similar to that used in $(2)[a, b] \subset M(\varphi)$ has the desired properties. Thus (3) is proved.

Now let $i$ be that member of $\{1,2, \cdots, n\}$ secured in (3). Let 2 be the smallest partition of $\beta_{i}(L)$ which contains $\beta_{i}\left(\varphi_{[\varphi]}\right)$. From (3) $\approx$ and $\beta_{i}\left(\mathscr{P}_{[\varphi \mid}\right)$ must coincide on $\left.\beta_{i}(a, b]\right)$. Thus $\beta_{i}(a)<\beta_{i}(b)$. Then $\beta_{i}(x) \leqq \beta_{i}\left(\phi^{1}(x)\right) \leqq \beta_{i}(a)<\beta_{i}\left(\phi^{0}(b)\right) \leqq \beta_{i}\left(\phi^{0}(y)\right) \leqq \beta_{i}(y)$.

Hence $\beta_{i}(x)$ and $\beta_{i}(y)$ must be in different elements of the partition $Q$. Thus we have $(x, y) \notin[\varphi] \vee\left[\beta_{i}\right]$. A fortiori $(x, y) \notin[\alpha] \vee\left[\beta_{i}\right]$ and our lemma is proved.

It is known (cf. [3]) the lattice of congruences for any lattice is distributive. For compact topological lattices we have

THEOREM 2.1. Suppose that $L$ is a compact distributive topological lattice of finite breadth. Then $\mathscr{C}(L)$ is a distributive lattice.

Proof. It is an immediate consequence of Lemma 2.1 that for each $[\alpha] \in \mathscr{L}(L),[\alpha] \vee \mathscr{C}(L)$ is a distributive lattice. We now claim that if $[\alpha] \in \mathscr{L}(L)$ then the map $[\varphi] \rightarrow[\alpha] \vee[\varphi]$ is a homomorphism of $\mathscr{C}(L)$ onto $[\alpha] \vee L$. Since this map obviously preserves joins we need only show that it also preserves meets. Suppose that $[\varphi]$ and [0] are members of $\mathscr{C}(L)$. Homomorphisms cannot raise breadth so $\operatorname{Br}(\varphi(L))=m \leqq B r(L) \geqq n=B r(\theta(L))$. From Theorem 3.1 of [2] we know that there is a set of $m$ elements of $\mathscr{L}(\varphi(L))$ which separates points in $\varphi(L)$ and a set of $n$ elements of $\mathscr{L}(\theta(L))$ which separates points in $\theta(L)$. This implies that there are $\left[\varphi_{1}\right],\left[\varphi_{2}\right], \cdots,\left[\varphi_{m}\right],\left[\theta_{1}\right]$, $\left[\theta_{2}\right], \cdots,\left[\theta_{n}\right] \in \mathscr{L}(L)$ such that $\bigwedge_{i=1}^{m}\left[\varphi_{i}\right]=[\varphi]$ and $\bigwedge_{i=1}^{m}\left[\theta_{i}\right]=[\theta]$. These facts and Lemma 2.1 enable us to obtain

$$
\begin{aligned}
{[\alpha] \vee([\varphi] \wedge[\theta]) } & =[\alpha] \vee\left(\left[\varphi_{1}\right] \wedge\left[\varphi_{2}\right] \wedge \cdots \wedge\left[\varphi_{m}\right] \wedge\left[\theta_{1}\right] \wedge\left[\theta_{2}\right] \wedge \cdots\left[\theta_{n}\right]\right) \\
& =\left([\alpha] \vee\left(\left[\varphi_{1}\right]\right) \wedge\left([\alpha] \wedge\left[\varphi_{2}\right]\right) \wedge \cdots \wedge\left([\alpha] \vee\left[\theta_{n}\right]\right)\right. \\
& =\left(\bigwedge_{i=1}^{m}\left([\alpha] \vee\left[\varphi_{i}\right]\right)\right) \wedge\left(\bigwedge_{i=1}^{n}\left([\alpha] \vee\left[\theta_{i}\right]\right)\right) \\
& =\left([\alpha] \vee\left(\bigwedge_{i=1}^{m}\left[\varphi_{i}\right]\right)\right) \wedge\left([\alpha] \vee\left(\bigwedge_{i=1}^{n}\left[\theta_{i}\right]\right)\right) \\
& =([\alpha] \vee[\varphi]) \wedge([\alpha] \vee[\theta]) .
\end{aligned}
$$

Hence the map $[\varphi] \rightarrow[\alpha] \vee[\varphi]$ is a homomorphism.

Next we shall show that the collection of homomorphisms $[\varphi] \rightarrow$ $[\alpha] \vee[\varphi]$ where $[\alpha] \in \mathscr{L}(L)$ separates points in $\mathscr{C}(L)$. Let $[\varphi],[\theta] \in$ $\mathscr{C}(L)$. Suppose that $[\varphi]=\bigwedge_{i=1}^{m}\left[\varphi_{i}\right]$ and $[\theta]=\bigwedge_{i=1}^{n}\left[\theta_{i}\right]$ are representa- 
tions of $[\varphi]$ and $[\theta]$ by elements of $\mathscr{L}(L)$ obtained as in the previous paragraph and suppose that no element in either representation is redundant. If $[\varphi] \neq[\theta]$ then we may assume that $[\varphi] \wedge[\theta] \neq[\theta]$. We claim that for some $i \in\{1,2, \cdots, m\},\left[\varphi_{i}\right] \vee[\varphi] \neq\left[\varphi_{i}\right] \vee[\theta]$. Suppose that this is not the case. Then for every $i \in\{1,2, \cdots, m\},\left[\rho_{i}\right]=$ $\left[\varphi_{i}\right]=\left[\varphi_{i}\right] \vee[\varphi]=\left[\varphi_{i}\right] \vee[\theta]$. Hence $\left[\varphi_{i}\right] \wedge[\theta]=[\theta]$ for every $i \epsilon$ $\{1,2, \cdots, m\}$. This allows us to conclude that $[\varphi] \wedge[\theta]=\left(\bigwedge_{i=1}^{n}\left[\varphi_{i}\right]\right) \wedge$ $[\theta]=[\theta]$ contrary to our assumption. Thus there are enough maps of the form $[\varphi] \rightarrow[\alpha] \vee[\varphi]$ where $[\alpha] \in \mathscr{L}(L)$ to separate points in $\mathscr{C}(L)$.

$\mathscr{C}(L)$ has enough homomorphisms onto distributive lattices to separate points. Thus $\mathscr{C}(L)$ can be embedded in a distributive lattice. Hence $\mathscr{C}(L)$ is distributive.

Note that our purpose in proving Theorem 2.1 is to aid in the proof of Theorem 2.2. No claims are made about the generality of Theorem 2.1.

THEOREM 2.2. Let $L$ be a compact, distributive topological lattice of finite breadth. Then $L$ has the congruence extension property.

Proof. Let $A$ be a closed sublattice of $L$ and let $[\varphi] \in \mathscr{C}(A)$. If $\operatorname{Br}(L)=1$ then $[\Phi]=\{(x, y)$ : there exists $a, b \in A$ with $\varphi(a)=\varphi(b)$ and $x, y \in[a, b]\} \cup \Delta$ is a congruence which is an extension of $[\varphi]$. Suppose that $\operatorname{Br}(L)=n \gg 1$. As a result of Theorem 3.1 of [2] $L$ can be embedded in a lattice $L^{\wedge}=C_{1} \times C_{2} \times \cdots \times C_{n}$ where each $C_{i}$ is a compact chain. Since this is the case we shall consider both $A$ and $L$ to be closed sublattices of $L^{\wedge}$ and proceed to show that $[\varphi]$ can be extended to a closed congruence on $L^{\wedge}$. Then a fortiori we will have proved that $[\varphi]$ can be extended to a closed congruence on $L$.

For each $i \in\{1,2, \cdots, n\}$ define $\pi_{i}$ to be the natural projection of $L^{\wedge}$ onto $C_{i}$ and $P_{i}$ to be the restriction of $\pi_{i}$ to $A$. Because $\mathscr{C}(A)$ is a distributive lattice and

$$
\bigwedge_{i=1}^{n}\left[P_{i}\right]=\Delta_{A},[\varphi]=[\varphi] \vee\left(\bigwedge_{i=1}^{n}\left[P_{i}\right]\right)=\bigwedge_{i=1}^{n}\left([\varphi] \vee\left[P_{i}\right]\right)
$$

Each $C_{i}$ is a chain and $P_{i}(A)$ is a closed sublattice of $C_{i}$ so the congruence on $P_{i}(A)$ associated with the natural map of $P_{i}(A)$ onto $A /$ $\left[P_{i}\right] \vee[\varphi]$ can be extended to a closed congruence $\left[\rho_{i}\right]$ on $C_{i}$. Consequently $\left[\rho_{i}^{\circ} \pi_{i}\right]$ is a member of $\mathscr{L}\left(L^{\wedge}\right)$ and it is also an extension of $[\varphi] \vee\left[P_{i}\right]$. We define $[\Phi]=\bigwedge_{i=1}^{n}\left[\rho_{i}^{\circ} \pi_{i}\right]$. Then

$$
[\Phi] \cap(A \times A)=\left(\bigwedge_{i=1}^{n}\left[\rho_{i}^{\circ} \pi_{i}\right]\right) \cap(A \times A)
$$




$$
\begin{aligned}
& =\bigwedge_{i=1}^{n}\left(\left[\rho_{i}^{\circ} \pi_{i}\right] \cap(A \times A)\right) \\
& =\bigwedge_{i=1}^{n}\left(\left[p_{i}\right] \vee[\varphi]\right) \\
& =[\varphi] .
\end{aligned}
$$

Thus $[\Phi]$ is an extension of $[\varphi]$.

3. Properties of $\mathbf{X}$. Let $I$ be the usual topological lattice on the closed real interval $[0,1]$. Let $K$ be the standard representation of the Cantor set in $I$ and let $N=\left\{1-1 / n ; n \in Z^{+}\right\} \cup\{1\}$. Then both $K$ and $N$ are closed sublattices of $I$. Let $X$ be the usual topological lattice on $\mathrm{X}_{i=1}^{\infty}\{0,1\}$. Define $W(X)$ to be the set of points of $X$ having only finitely many nonzero coordinates.

Recall that the set of local minima of $X$ is dense in $X$ [6]. A sequence $\left\{x_{n} ; n \in \omega\right\}$ is nondecreasing if $x_{n} \wedge x_{n+1}=x_{n}$ for all $n \in \omega$.

LEmma 3.1. Let $x \in X$ and let $w \in W(X)$. Then the following statements hold:

(1) $w \wedge X$ is finite.

(2) There is a nondecreasing sequence in $(x \wedge X) \cap W(X)$ which converges to $x$.

(3) $W(X)$ is the set of local minima of $X$. Hence $W(X)$ is dense in $X$.

Proof. (1) is obvious and (3) is a direct consequence of (1) and (2). Thus it only remains to prove (2). For $x \in X$ we define a sequence $\left\{w_{n} ; n \in \omega\right\}$ by having $w_{n}$ agree with $x$ for the first $n$ coordinates and thereafter having all coordinates zero. Then each $w_{n} \in$ $W(X)$ and $\left\{w_{n} ; n \in \omega\right\}$ obviously converges to $x$.

Following [5] we say that a continuous homomorphism $\varphi$ from a compact semilattice $S$ onto a semilattice $T$ has full cross-section if there is a closed sub-semilattice $A$ of $S$ such that $\varphi$ restricted to $A$ is an isomorphism of $A$ onto $T$.

LEMMA 3.2. Let $\varphi$ be a continuous meet-homomorphism of $X$ onto the compact zero-dimensional chain $C$. Then $C$ is the continuous meet-homomorphic image of $N$. Moreover, $\varphi$ has full cross-section.

Proof. Let $B$ be the set of local minima of $C$ and let $A=\{x \in$ $X ; x$ is the least element of $\varphi^{-1}(b)$ for some $\left.b \in B\right\} . A$ is a chain and from Lemma 3.1, $A \subseteq W(X)$. Then since $B^{*}=C$ we have $\varphi\left(A^{*}\right)=$ $C$. In view of Lemma 3.1, $A^{*}$ must be the continuous meet-homomorphic image of $N$ and $A^{*}$ must define a full cross-section for $\varphi$. 
THEOREM 3.1. If there is a continuous meet-homomorphism of $X$ onto $Y$, then $Y$ is zero-dimensional.

Proof. Suppose that $\phi$ is a continuous meet-homomorphism from $X$ onto a semilattice $S$ where $\operatorname{dim} S \geqq 1$. Since $\operatorname{dim} S \geqq 1, S$ must possess a nondegenerate component $S_{0}$. $S_{0}$ is a compact semilattice so it has a least element $s_{0}$. Choose $s \in S \backslash\left\{s_{0}\right\}$. The Rees-quotient $s S / s_{0} S$ is a nondegenerate connected semilattice and it is the continuous meet-homomorphic image of $X$. As a consequence of Theorems 2.1, 3.1 and 4.3 of $] 7], s S / s_{0} S$, and hence also $X$, must have a continuous meet-homomorphism onto $I$. Let $\eta$ be one such homomorphism. $w \wedge$ $X$ is finite for all $w \in W(X)$. Hence $\eta(W(X))=0$. This implies that $\eta\left(W(X)^{*}\right)=0$. However, $X=W(X)^{*}$. Thus we have arrived at a contradiction. Therefore our theorem is proved.

We now show that $X$ does not have the congruence extension property. In fact, we can say more.

CoRollary 3.1. There is a linearly ordered sublattice $K$ of $X$ and a closed congruence $\zeta$ on $K$ such thxt $\zeta$ cannot be extended to a closed meet-congruence on $X$.

Proof. From Theorem 2 of [8] $K$ can be embedded in $X$. Thus we may consider $K$ to be a sublattice of $X$. On $K$ the relation defined by identifying the end points of complementary intervals is a member of $\mathscr{C}(K)$. Moreover $\zeta(K)$ is a compact, connected lattice. From Theorem 3.1 it follows that [ $\zeta$ ] cannot be extended to a closed meet-congruence on $X$.

We now provide several examples which are variants of the example used in Corollary 3.1.

Example 1. Let $C$ denote the image of $K$ in $X$ under an embedding $\rho$ where $\rho(0)=0$ and $\rho(1)=1$. $X$ can be considered to be a sublattice of $I^{\omega}$. Extend $C$ to a maximal chain $J$ in $I^{\omega}$. The closed

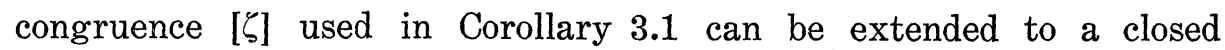

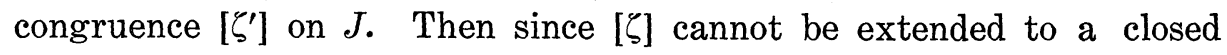
meet-congruence on $X$ it follows that $\left[\zeta^{\prime}\right]$ cannot be extended to a closed meet-congruence on $I^{\omega}$.

Example 2. In the previous example let $S=\cup\{x \wedge J ; x \in X\}$. Then $S$ is a compact, one-dimensional semilattice and $J$ is the unique thread from the zero of $S$ to the identity of $S$. Applying the reasoning used in Example 1 the congruence [ $\left.\zeta^{\prime}\right]$ on $J$ cannot be extended to $S$. $S$ has the additional property that $J$ cannot be the continuous 
(semi-lattice) homomorphic retract of $S$. Note that this will remain true if $I$ is replaced by any standard thread.

\section{REFERENCES}

1. L. W. Anderson, One dimensional topological lattices, Proc. Amer. Math. Soc., 10 (1959), 715-720.

2. K. A. Baker and A. R. Stralka, Compact, distributive lattices of finite breadth, Pacific J. Math., 34 (1970), 311-320.

3. N. Funayama and T. Nakayama, On the distributivity of a lattice of lattice-congruences, Proc. Imp. Acad. Tokyo, 18 (1942), 553-554.

4. G. Gratzer, Lectures on Lattice Theory, Volume 1, W. H. Freeman \& Co., San Francisco (to appear).

5. R. P. Hunter and L. W. Anderson, Certain homomorphisms of a compact semigroup onto a thread, J. Austr. Math. Soc., 7 (1967), 311-322.

6. R. J. Koch, Arcs in partially ordered spaces, Pacific J. Math., 9 (1959), 723-728.

7. J. D. Lawson. Topological semilattices with small semilattices, J. London Math. Soc., (2), 1 (1969), 719-724.

8. K. Numakura, Theorems on compact totally disconnected semigroups and lattices, Proc. Amer. Math. Soc., 8 (1957), 623-626.

Received October 26, 1970 and in revised form March 9, 1971. Supported in part by NSF Grant GP-18832.

University of CAlifornia, Riverside 


\title{
PACIFIC JOURNAL OF MATHEMATICS
}

\author{
EDITORS
}

\author{
H. Samelson \\ Stanford University \\ Stanford, California 94305 \\ C. R. HOBBY \\ University of Washington \\ Seattle, Washington 98105
}

J. DugundJI

Department of Mathematics

University of Southern California

Los Angeles, California 90007

RICHARD ARENS

University of California

Los Angeles, California 90024

\section{ASSOCIATE EDITORS}

E. F. BECKENBACH
B. H. NEUMANN

F. WoLF
K. YosHIDA

\section{SUPPORTING INSTITUTIONS}

\author{
UNIVERSITY OF BRITISH COLUMBIA \\ CALIFORNIA INSTITUTE OF TECHNOLOGY \\ UNIVERSITY OF CALIFORNIA \\ MONTANA STATE UNIVERSITY \\ UNIVERSITY OF NEVADA \\ NEW MEXICO STATE UNIVERSITY \\ OREGON STATE UNIVERSITY \\ UNIVERSITY OF OREGON \\ OSAKA UNIVERSITY \\ UNIVERSITY OF SOUTHERN CALIFORNIA
}

\author{
STANFORD UNIVERSITY \\ UNIVERSITY OF TOKYO \\ UNIVERSITY OF UTAH \\ WASHINGTON STATE UNIVERSITY \\ UNIVERSITY OF WASHINGTON \\ $\stackrel{*}{*} \stackrel{*}{*} \stackrel{*}{*}{ }^{*}{ }^{*}$ MERICAN MATHEMATICAL SOCIETY \\ CHEVRON RESEARCH CORPORATION \\ NAVAL WEAPONS CENTER
}

The Supporting Institutions listed above contribute to the cost of publication of this Journal, but they are not owners or publishers and have no responsibility for its content or policies.

Mathematical papers intended for publication in the Pacific Journal of Mathematics should be in typed form or offset-reproduced, (not dittoed), double spaced with large margins. Underline Greek letters in red, German in green, and script in blue. The first paragraph or two must be capable of being used separately as a synopsis of the entire paper. The editorial "we" must not be used in the synopsis, and items of the bibliography should not be cited there unless absolutely necessary, in which case they must be identified by author and Journal, rather than by item number. Manuscripts, in duplicate if possible, may be sent to any one of the four editors. Please classify according to the scheme of Math. Rev. Index to Vol. 39. All other communications to the editors should be addressed to the managing editor, Richard Arens, University of California, Los Angeles, California, 90024.

50 reprints are provided free for each article; additional copies may be obtained at cost in multiples of 50 .

The Pacific Journal of Mathematics is published monthly. Effective with Volume 16 the price per volume (3 numbers) is $\$ 8.00$; single issues, $\$ 3.00$. Special price for current issues to individual faculty members of supporting institutions and to individual members of the American Mathematical Society: $\$ 4.00$ per volume; single issues $\$ 1.50$. Back numbers are available.

Subscriptions, orders for back numbers, and changes of address should be sent to Pacific Journal of Mathematics, 103 Highland Boulevard, Berkeley, California, 94708.

PUBLISHED BY PACIFIC JOURNAL OF MATHEMATICS, A NON-PROFIT CORPORATION

Printed at Kokusai Bunken Insatsusha (International Academic Printing Co., Ltd.), 7-17, Fujimi 2-chome, Chiyoda-ku, Tokyo, Japan. 


\section{Pacific Journal of Mathematics}

J. T. Borrego, Haskell Cohen and Esmond Ernest Devun, Uniquely

representable semigroups on the two-cell .................. 565

Glen Eugene Bredon, Some examples for the fixed point property ........ 571

William Lee Bynum, Characterizations of uniform convexity .......... 577

Douglas Derry, The convex hulls of the vertices of a polygon of order $n \ldots 583$

Edwin Duda and Jack Warren Smith, Reflexive open mappings .......... 597

Y. K. Feng and M. V. Subba Rao, On the density of $(k, r)$ integers ........ 613

Irving Leonard Glicksberg and Ingemar Wik, Multipliers of quotients of

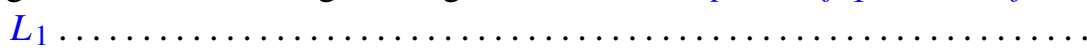

John William Green, Separating certain plane-like spaces by Peano

continua.........................................

Lawrence Albert Harris, A continuous form of Schwarz's lemma in normed

linear spaces .................................... 635

Richard Earl Hodel, Moore spaces and $w$-spaces ............... 641

Lawrence Stanislaus Husch, Jr., Homotopy groups of PL-embedding spaces.

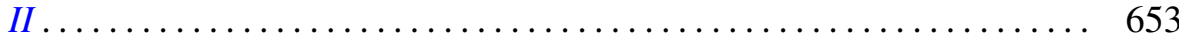

Yoshinori Isomichi, New concepts in the theory of topological

space-supercondensed set, subcondensed set, and condensed set.....

J. E. Kerlin, On algebra actions on a group algebra .................

669

Keizō Kikuchi, Canonical domains and their geometry in $C^{n} \ldots \ldots \ldots \ldots 681$

Ralph David McWilliams, On iterated $w^{*}$-sequential closure of cones. .

697

C. Robert Miers, Lie homomorphisms of operator algebras ..

717

Louise Elizabeth Moser, Elementary surgery along a torus knot ...

737

Hiroshi Onose, Oscillatory properties of solutions of even order differential

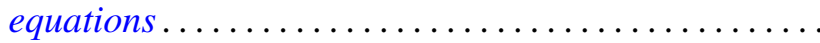

747

Wellington Ham Ow, Wiener's compactification and $\Phi$-bounded harmonic

functions in the classification of harmonic spaces...

Zalman Rubinstein, On the multivalence of a class of meromorphic

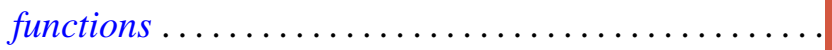

771

785

Hans H. Storrer, Rational extensions of modules

795

topological lattices ...........................

Robert Evert Stong, On the cobordism of pairs .................. 803
Albert Leon Whiteman, An infinite family of skew Hadamard matrices . . . 817

Lynn Roy Williams, Generalized Hausdorff-Young inequalities and mixed

norm spaces 